\title{
Multiple Intra-Tube Junctions in the Inner Tube of Peapod-Derived Double Walled Carbon Nanotubes: Theoretical Study and Experimental Evidence
}

\author{
Ziwei Xu ${ }^{\text {a }}$, Hui Li ${ }^{* b}$, Kazunori Fujisawa ${ }^{c}$, Yoong Ahm Kim ${ }^{c}$, Morinobu Endo ${ }^{c}$, Feng Ding ${ }^{* a}$ \\ ${ }_{5}$ Received (in $\left.X X X, X X X\right)$ Xth $X X X X X X X X X 20 X X$, Accepted Xth XXXXXXXXX 20XX \\ DOI: 10.1039/b000000x
}

The coalescence process of fullerenes in the hollow core of single wall carbon nanotube is systematically explored by kinetic Monte Carlo method. Two elongation (or growth) modes via the coalescence (i) between an inner tube and fullerenes and (ii) between neighboring inner tubes are 10 identified. It is found that the coalescence of two inner tubes mostly creates a very stable intra-tube junction which is composed of multiple pentagon-heptagon pairs. As a consequence, the study predicts that the inner tube of peapod-grown double walled carbon nanotubes (DWNTs) must contain many intratube junctions. Careful high resolution transmission electron microscopy observation on peapod-grown DWNT sample provides an experimental evidence on the presence of the junctions.

\section{1. Introduction}

Carbon peapods, the hybrids of two important carbon nanomaterials (i.e., carbon nanotubes (CNTs) and fullerenes), have drawn great attention since their first experimental synthesis in $1998 .^{1-7}$ It is amazing that the coalescence of the encapsulated 20 fullerenes leads to the formation of uniform inner tube and then transforms a peapod into a double-walled CNT (DWNT). ${ }^{8,9}$ After 10-year extensive study, "peapod" method becomes a standard way of synthesizing DWNTs. Compared to other synthetic methods, "peapod" method has several advantages: (i) The 25 process is free of catalyst; (ii) It can provide small-diameter DWNT sample; ${ }^{10}$ (iii) The products have a very high DWNT to SWNT ratio. ${ }^{11}$

The theoretical study of the coalescence of encapsulated fullerenes has been a great challenge for many years. ${ }^{8,} 12-24,25 \mathrm{In}$ 30 terms of the methods used, the theoretical studies previously published can be classified into three categories:

Molecular dynamics (MD) simulation, including both classical $\mathrm{MD}$ and quantum $\mathrm{MD}$, is one of the most common methods. ${ }^{13,26-}$

${ }^{31}$ It's very unfortunate that none of previous MD simulations has

35 been able to end up with a well-defined chirality of inner tube and thus the information about the peapods derived DWNTs is incomplete. This is due to the limited time scale of MD simulation. The time scale for classical MD and for quantum MD is ns and ps, respectivley whereas the experimental time is 40 ranging from minutes to hours. Their difference is more than 12 orders of magnitude.

Kinetic simulations based on C-C bond rotation or so called the generalized Stone-Wales (GSW) transformation have been widely used to simulate the coalescence process of fullerenes. ${ }^{32}$

45 The kinetic simulations are very diverse compared to the MD simulation. In 2002, Zhao and coworkers have discovered a manual bond rotation procedure to transform a $\mathrm{C}_{60}$ dimer into a short $\mathrm{C}_{120} \mathrm{SWNT}^{33}$ and then the method has been applied to study the welding between two SWNTs and between a SWNT and a 50 fullerene. ${ }^{34,35}$ This manual process is very time consuming and thus can only be used to explore a very limited number of samples. Furthermore this procedure is guided by several geometrical rules, which is very different from the real driving force in a natural process, the change of energy and the energy 55 barrier. Latterly, the transition state barrier of every bond rotation has been calculated by Han et al.. ${ }^{36}$

In 2006, the action-derived molecular dynamics (ADMD) method has been introduced to simulate the fullerene coalescence process by Lee and coworkers. ${ }^{37}$ The energy difference is the 60 driving force in a ADMD simulation and thus the ADMD simulation is able to produce a reasonable low energy path for fullerene coalescence. While, same as the manual work, the final structure of the ADMD simulation is predetermined. Thus the information about inner tube structure, e.g., the chiral angle or 65 diameter correlation between the inner and outer tubes, can't be retrieved.

We recently have developed an energy driven kinetic Monte Carlo (EDKMC) method to study the structural transformation of nano carbon materials. ${ }^{38-41}$ In a EDKMC trajectory, the energy 70 change of a C-C bond rotation is used as the driving force. The EDKMC method has been successfully applied to simulate the coalescence of two fullerenes in a SWNT recently. ${ }^{38}$ The study shows that there is no chiral angle correlation between the inner and outer tubes and the initial inner tube always tends to have a 75 large chiral angle which is near 30 degree. $^{38}$ The agreement between these theoretical predictions and experimental measurements validated the EDKMC method. ${ }^{38}$ Beyond, previous study has shown that the space constrain of outer tube plays a crucial role in the inner tube formation. The final inner tube 

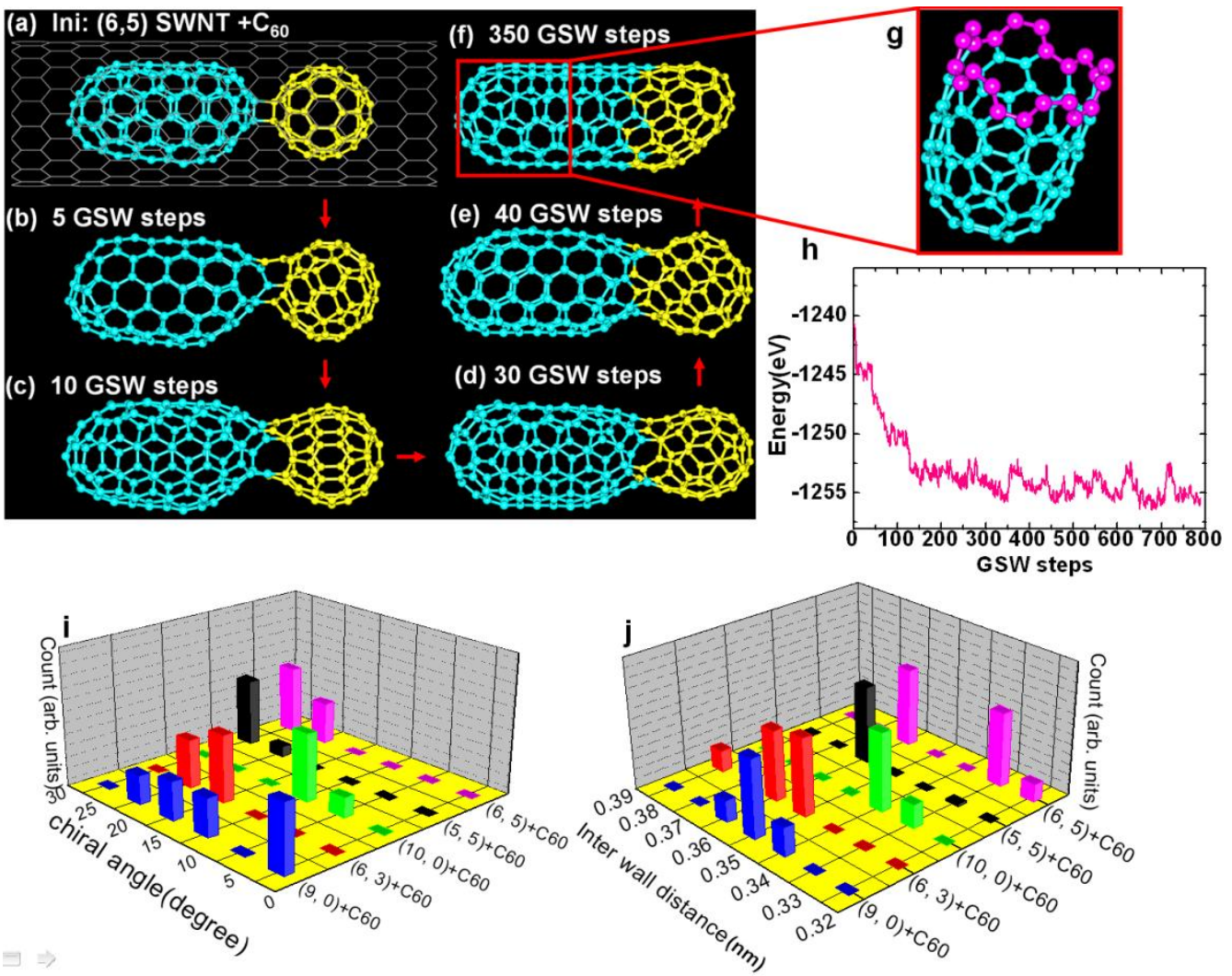

Fig. 1 (a-f) Snapshots in a coalescence of a $(6,5) \mathrm{C}_{120}$ tube $+\mathrm{C}_{60}$. $(\mathrm{g}) \mathrm{A}$ magnified inner wall tube stem from snapshot (f), from which the chirality of the 5 inner tube can be clearly identified. (h) The energy evolution during the fusion of $(6,5) \mathrm{C}_{120}+\mathrm{C}_{60}$. In these EDKMC snapshots, the outer (18,0) tube is only shown in the initial configuration (a) for clarity. (i) The chiral angle distributions of the inner wall tubes obtained in the coalescences of different $\mathrm{C}_{120}$ tubes $+\mathrm{C}_{60}$ (five $\mathrm{C}_{120}$ tubes form from armchair to zigzag, (5,5), $(6,5),(6,3),(9,0)$ and $(10,0)$, are explored). (j) The distribution of the wall-wall distances between the final inner tubes and the $(18,0)$ outer tube.

10 observed wall-wall distance of DWNTs. ${ }^{42}$

In this article, we present a systematic EDKMC study of inner tube elongation. Two processes of inner tube elongation, namely inner tube $+\mathrm{C}_{60}$ coalescence and inner tube + inner tube coalescence are identified. For the inner tube $+\mathrm{C}_{60}$ coalescence, 15 most inner tubes tend to maintain its original chirality and the longer the tube stem is, the robuster its chirality is. For the inner tube + inner tube process, an inner tube with a longer chirality identified stem has larger probability for its chirality to be inherited. An intra-tube junction, which is composed of multiple 20 pentagon-heptagon pairs $(5 \mid 7 \mathrm{~s})$, will be formed between two longer inner tubes. This study reveals that the inner tube of a peapod-derived DWNT must contain highly concentrated intratube junctions in the inner wall. Furthermore, careful high resolution transmission electronic microscopy (HRTEM) 25 observation was carrid out in order to support both the tube elongation processes and the formation of the intra-tube junctions.
The EDKMC method is used to study the elongation process of short inner tubes. Denoting the energy of the relaxed initial 30 structure as $E_{\mathrm{i}}$, the energy of final structure which is obtained by rotating a randomly selected $\mathrm{C}-\mathrm{C}$ bond of the initial structure by 90 degree as $E_{f}$. Similar to the standard Monte Carlo simulation, the probability of accepting the final structure is 1 if $\triangle E=E_{\mathrm{f}}$ $E_{\mathrm{i}}<0$ or $\exp (-\triangle E / k T)$ if $\triangle E>0$, where $k$ is the Boltzmann 35 constant and $T$ is the temperature. More details of the EDKMC method and its validity are shown in previous publication. ${ }^{38}$ In previous studies, we have shown that there's no tube chiral angle correlation between the inner wall tube and outer wall tube and thus a $(18,0)$ SWNT was chosen as the only outer wall tube in all 40 trajectories. The Monte Carlo temperature of all trajectories was set as $2000 \mathrm{~K}$, which is close to the experimental temperature of deriving peapods into DWNTs.

For the popular $\mathrm{C}_{60} \mathrm{~s} @ \mathrm{SWNT}$ peapods, the formation of peapods derived DWNT starts with a $\mathrm{C}_{60}$ dimer. The fusion of a ${ }_{45} \mathrm{C}_{60}$ dimer resulting in a short $\mathrm{C}_{120}$ SWNT, $\mathrm{C}_{60}+\mathrm{C}_{60} \rightarrow \mathrm{C}_{120}$

\section{Simulation Method}




\section{Cite this: DOI: 10.1039/c0xx00000x}
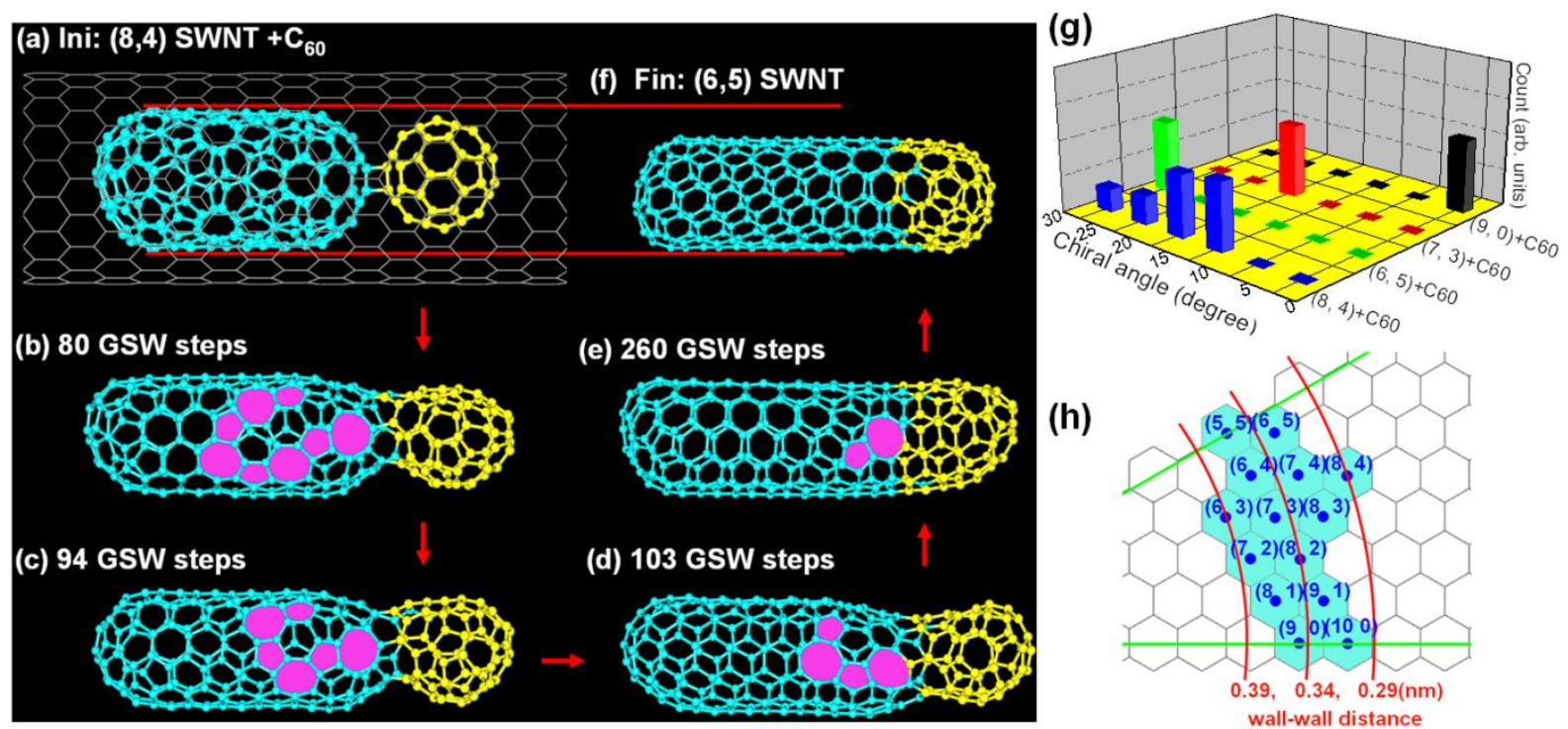

Fig. 2 Panels (a-f) are the snapshots taken in the fusion of a $(8,4) \mathrm{C}_{180}$ inner tube and a $\mathrm{C}_{60}$. The final inner tube (f) has smaller diameter than that of the original one (a). (g) The chiral-angle distributions of the inner tubes which are formed in the fusion of different $\mathrm{C}_{180}$ inner tubes with $\mathrm{C}_{60}$. (h) The wall-wall distances between various inner tubes and the outer $(18,0)$ tube.

${ }_{5}$ SWNT, has been extensively studied before.$^{38}$ Here we consider the inner tube elongation from a short $\mathrm{C}_{120}$ SWNT. In the elongation process, if a $\mathrm{C}_{120}$ is surrounded by $\mathrm{C}_{60}$ s, the next step must be the fusion of the $\mathrm{C}_{120}$ with a nearby $\mathrm{C}_{60}, \mathrm{C}_{120}+\mathrm{C}_{60} \rightarrow$ $\mathrm{C}_{180}$, and then, $\mathrm{C}_{180}+\mathrm{C}_{60} \rightarrow \mathrm{C}_{240} \ldots$ Beyond the continuous 10 growth of an inner tube by adsorbing $\mathrm{C}_{60} \mathrm{~s}$, the meeting of two tubes and their fusion is another important process towards inner tube elongation, e.g., $\mathrm{C}_{120}+\mathrm{C}_{120}, \mathrm{C}_{120}+\mathrm{C}_{180}$ or $\mathrm{C}_{180}+\mathrm{C}_{180}$.

So, in order to achieve a comprehensive understanding of the elongation process, we use $\mathrm{C}_{120} \mathrm{~s}$ and $\mathrm{C}_{180} \mathrm{~s}$ to represent short tube 15 and long inner tube, respectively and designed five different inner tube elongation processes, which are (i) $\mathrm{C}_{120}+\mathrm{C}_{60}$; (ii) $\mathrm{C}_{180}+\mathrm{C}_{60}$; (iii) $\mathrm{C}_{120}+\mathrm{C}_{120}$; (iv) $\mathrm{C}_{120}+\mathrm{C}_{180}$ and (v) $\mathrm{C}_{180}+\mathrm{C}_{180}$, respectively.

In all simulations, the initial $\mathrm{C}_{120} \mathrm{~s}$ are obtained by the coalescence of two $\mathrm{C}_{60} \mathrm{~S}$ and $\mathrm{C}_{180} \mathrm{~s}$ are formed in the 20 coalescence of $\mathrm{C}_{120}+\mathrm{C}_{60}$. Certainly, the fusion of further longer tubes (e.g., $\mathrm{C}_{240}$ ) can be considered as well. But as we can see, the robusticity of the $\mathrm{C}_{180}$ inner tubes ensures that it's not necessary to consider longer inner tubes beyond $\mathrm{C}_{180}$.

\section{Results}

\section{${ }_{25} 3.1$ The elongation of short inner tubes: $\mathbf{C}_{120}+\mathbf{C}_{60}$}

Fig. 1a-f depict the elongation of a short $(6,5)$ SWNT by adsorbing a $\mathrm{C}_{60}$. The EDKMC simulation starts with an initial configuration that the short SWNT is linked to the $\mathrm{C}_{60}$ by two C$\mathrm{C}$ covalent bonds, which can be viewed as an extreme narrow 30 neck (Fig. 1a). During the initial stage, most GSW bond rotations occur around the neck area because of fast relaxation of the high curvature energy. The curvature energy drops notably (Fig. 1h) and the neck becomes thicker and thicker (Fig. $1 \mathrm{a} \rightarrow \mathrm{b} \rightarrow \mathrm{c} \rightarrow \mathrm{d} \rightarrow \mathrm{e}$ ). Eventually, a perfect longer $(6,5)$ tube was formed after about 35350 GSW steps (Fig. 1f and Fig. 1g). After that, the energy only fluctuates in a very small amplitude (Fig. 1h) and the stem of the tube remains unchanged. In this trajectory, the chirality identified stem of the short SWNT is maintained and thus the final inner tube inherits the original chirality of the short tube. This ${ }_{40}$ simulation presents the robusticity of the short inner tubes.

In order to further test the robusticity of inner tubes, the coalescences of five different $C_{120}$ tube isomers, which are $(6,5)$, $(5,5),(10,0),(6,3)$ and $(9,0)$ tubes, with a $\mathrm{C}_{60}$ are systematically studied. 20 40 EDKMC successful trajectories were run for 45 every combination (details to see Table $\mathrm{S} 1^{\dagger}$ ). The statistics over the final inner tubes' chiral angles and wall-wall distances are shown in Fig. $1 \mathrm{i}$ and $1 \mathrm{j}$. It can be clearly seen that not all tubes maintain their original chiralities in the EDKMC trajectories. For example, more than half of the final tubes obtained from the ${ }_{50}$ coalescences of $(9,0)$ inner tube with $\mathrm{C}_{60}$ are chrial tubes. In contrast, short inner tubes with large chiral angles tend to maintain their original chiralities, e.g., $83 \%$ of the $(5,5)$ tubes remain the same after the fusion with a $\mathrm{C}_{60}$. The robusicity of large chiral angle tubes is in agreement with what is shown in ${ }_{55}$ previous study. ${ }^{38}$

\subsection{The elongation of long inner tubes: $C_{180}+C_{60}$}

Fig. 2a-f are snapshots taken in the coalescence of a $(8,4) \mathrm{C}_{180}$ inner tube and a $\mathrm{C}_{60}$. During the coalescence, several pentagonheptagon pairs (5|7s) are excited from the left end of the $\mathrm{C}_{180}$ tube ${ }_{60}$ and these $5 \mid 7 \mathrm{~s}$ gradually propagate through the tube wall to the tube- $\mathrm{C}_{60}$ neck area and sink there $(\mathrm{b} \rightarrow \mathrm{c} \rightarrow \mathrm{d})$. The final inner tube, whose chirality is $(6,5)$, is notably smaller than the original tube 


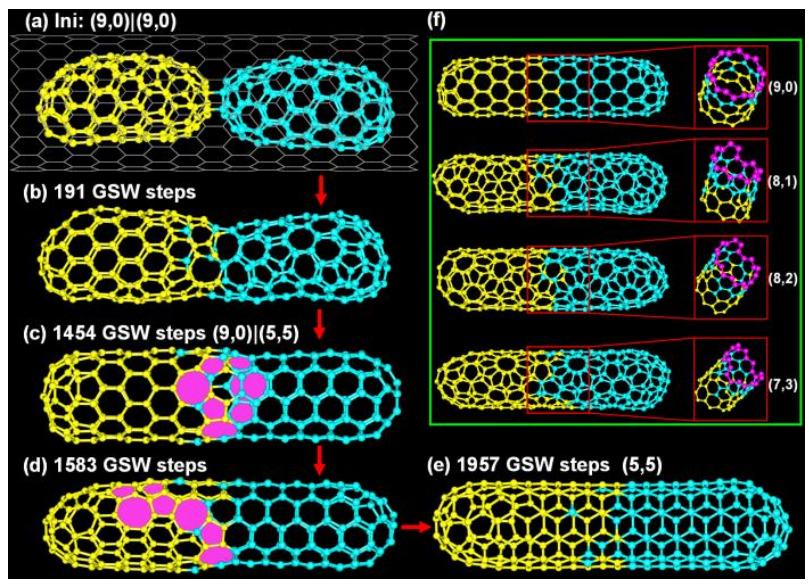

Fig. 3. (a-e) Snapshots in the fusion of two $(9,0) C_{120}$ inner tubes into a $(5,5) \quad C_{240}$ tube. (f) Five different $C_{240}$ tubes observed in different trajectories.

5 (Fig. 2f). This indicates that the wall-wall distance between the inner and outer tubes is a crucial parameter that controls the robusticity of the inner tube. The wall-wall distance between the $(8,4)$ inner tube and the $(18,0)$ outer tube is only $0.29 \mathrm{~nm}$ (Fig. $2 \mathrm{~h}$ ), which is $18 \%$ smaller than the equilibrium layer-layer 10 distance in graphite, $\sim 0.34 \mathrm{~nm}$. On the other hand, the final $(6,5)$ tube, which has a wall-wall distance of $0.355 \mathrm{~nm}$, fit the $(18,0)$ tube perfectly.

Except the large diameter $(8,4) \mathrm{C}_{180}$ inner tube, other three inner tubes, $(9,0),(7,3)$ and $(6,5)$, explored in this study have well 15 fitted wall-wall distances with the $(18,0)$ outer tube, which are $0.352 \mathrm{~nm}, 0.331 \mathrm{~nm}$ and $0.355 \mathrm{~nm}$, respectively. As a consequence, every trajectory (see Table $S 2^{\dagger}$ ) results in a tube with exact same chirality as the original one's (Fig. $2 \mathrm{~g}$ ). The apparent contrast with the results shown in Fig. 1 indicates that an inner tube with a 20 chirality identified stem of $2 \mathrm{~nm}$ or longer is very robust and its chirality is very hard to be changed unless its wall-wall distance with the outer tube is far from the equilibrium distance, $0.34 \mathrm{~nm}$.

3.3 The competition between two short tubes: $\mathbf{C}_{\mathbf{1 2 0}}+\mathbf{C}_{\mathbf{1 2 0}}$

Here we focus on the fusion of two short inner tubes, $\mathrm{C}_{120}+$

${ }_{25} \mathrm{C}_{120}$. Fig. 3a-e are representative snapshots during the fusion of two $(9,0) C_{120}$ tubes. At the initial elongation stage, the inner tube on the right side becomes very defective and then it turns into a $(5,5)$ tube (Fig. $3 a \rightarrow b \rightarrow c$ ). A intra-tube junction, which contains a few $5 \mid 7 \mathrm{~s}$ and connects the $(9,0)$ tube on the left and the newly 30 formed $(5,5)$ tube on the right, is formed during the process (Fig. $3 \mathrm{c})$. In the following simulation, the $5 \mid 7$ pairs around the intratube junction gradually glide to the left side of the inner tube and disappeared into the tube cap eventually (Fig. $3 c \rightarrow d \rightarrow$ e). The full EDKMC trajectory costs about 2000 GSW steps, which is about 35 one order of magnitude slower than the fusion of $\mathrm{C}_{120}+\mathrm{C}_{60}$. The long trajectory implies that the fusion of two short tubes into a single chirality one is very difficult and the diffusion of a tubetube junction is very slow.

It's important to note that the final tubes can be very diverse. 40 Beside the before mentioned $(5,5)$ tube, other chiral tubes, for example $(9,0),(8,1),(8,2),(7,3)$ tubes, have been observed in EDKMC trajectories (see Fig. 3f).

3.4 The competition between a longer inner tube and a shorter inner tube: $\mathbf{C}_{180}+\mathbf{C}_{120}$

45 From the results presented above, we can conclude that these very short $\mathrm{C}_{120}$ tubes cannot maintain their chiralities during the fusions of $\mathrm{C}_{120}+\mathrm{C}_{60}$ or $\mathrm{C}_{120}+\mathrm{C}_{120}$ but the longer $\mathrm{C}_{180}$ tubes are very robust. So it's very natural to suspect that a longer tube is going to beat a short one to inherit its gene, the chirality, in the 50 fusion of $\mathrm{C}_{180}+\mathrm{C}_{120}$. Fig. 4 visibly shows such a trend, the stem of the $(9,0)$ tube becomes longer and longer and eventually dominates the final tube in the coalescence of a $(9,0) \mathrm{C}_{180}$ tube + $(6,5) \mathrm{C}_{120}$ tube. This indicates that the tube length is the most critical parameter in the tube-tube fusion.

${ }_{55}$ 3.5 The competition between two longer tubes: $C_{180}+C_{180}$

So far, we can conclude that it's very hard to change the chirality of an inner tube whose chirality identified stem is longer than $2 \mathrm{~nm}$. Therefore, what will happen if two long $\mathrm{C}_{180}$ inner tubes meet each other and fuse into one? The fusion process of a ${ }_{60}(5,5) \mathrm{C}_{180}$ tube and a $(9,0) \mathrm{C}_{180}$ tube is shown in Fig. 5. A tubetube neck which has nearly same diameter as the original tubes is formed in between after $\sim 500 \mathrm{GSW}$ annealing steps $(\mathrm{a} \rightarrow \mathrm{b} \rightarrow \mathrm{c})$. An intra-molecular ring of defects that is composed of a few $5 \mid 7$

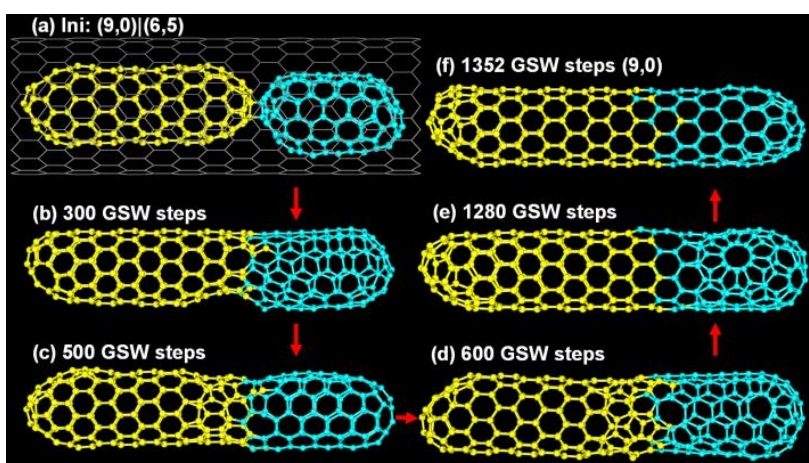

65

Fig. 4 The fusion of a $(9,0) C_{180}$ tube $+(6,5) C_{120}$ tube

pairs can be identified around the neck area (c). The position of the ring does not change in the following long EDKMC simulation (up to $3000 \mathrm{GSW}$ steps) though the arrangement of the $5 \mid 7 \mathrm{~s}$ becomes different $(c \rightarrow d)$. The robusiticity of the two inner 70 tubes results in a stable intra-tube junction. To link two different SWNTs into one, such a junction is inevitable. Similar intra-tube junctions are seen in other trajectories. As shown in Fig. 5f, the junctions of $(9,1)|(9,0),(7,3)|(9,0)$ and $(6,4) \mid(9,0)$ contains 1,3 and $45 \mid 7$ pairs, respectively.

\section{4. Discussion}

\subsection{The intra-tube junction as a rolled up graphene grain} boundary

As shown in the perspective view of the intra-tube junction between a $(5,5)$ tube and a $(9,0)$ tube (Fig. 5e), the $5 \mid 7$ pairs form 80 a ring of defects in a regular manner, the pentagon of a $5 \mid 7$ pair contacts with the heptagon of a neighboring pair or pentagons and heptagons appear alternatively along the tube circumference. The novel ring formation is a consequence of minimizing the junction's formation energy. Recent study in graphene grain 85 boundary reveals that linear arrangement of $5 \mid 7$ pairs minimizes the boundary formation energy because of the cancellation of the compression and tension strains around a $5 \mid 7$ pair. ${ }^{43} \mathrm{~A}$ carbon nanotube can be viewed as a rolled up graphene and therefore the ring of $5 \mid 7 \mathrm{~s}$ can be viewed as a rolled up graphene grain boundary. $90 \quad$ Fig. $5 \mathrm{~g}$ shows the number of $5 \mid 7$ pairs of an intra-tube junction 

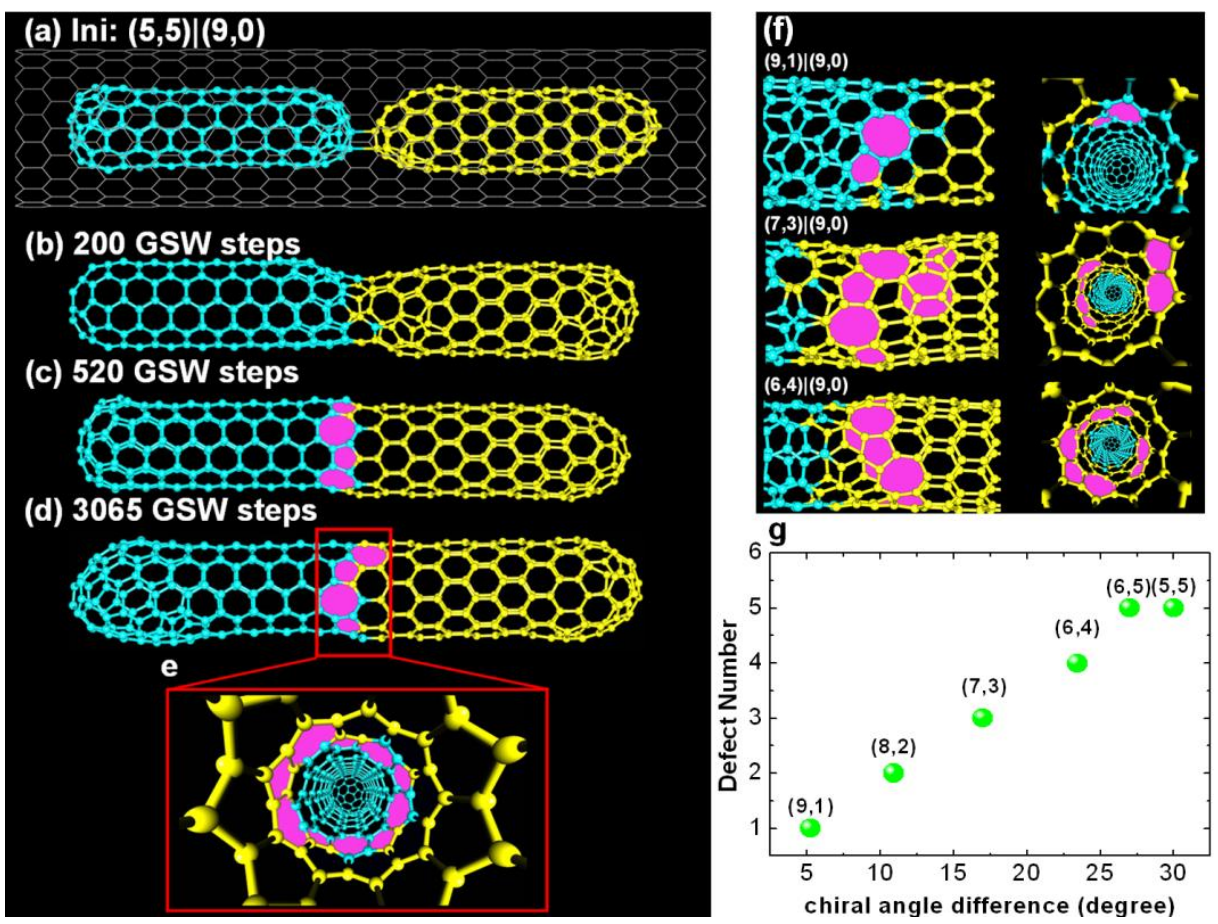

Fig. 5 (a-d) Some typical snapshots during the coalescence of a $(9,0) C_{180}$ inner tube and a $(5,0) C_{180}$ inner tube. (e) The perspective view of the tube-tube junction. (f) Several other tube-tube junctions formed in the coalescence of a $(9,0) C_{180}$ tube and a few other $C_{180}$ tubes. (g) The number of $5 \mid 7$ pairs at the tube-tube junction as a function of chiral angle difference between two tubes.

5 as a function of chiral angle difference between the two tubes. A clear linear relationship, $N_{5 \mid 7} \sim \Delta \theta$, is presented. Such a behavior is a bit surprising since it has been theoretically proved that only one pentagon and one heptagon are sufficient to connect two arbitrary SWNTs (Fig. 6a $\rightarrow$ b). ${ }^{44,45}$ It's important to note that the ${ }_{10}$ previous theory allows the hybrid tube wall to turn a sharp angle at the junction (Fig. 6b). While, a peapod derived inner tube is constrained inside a straight outer tube and thus has to maintain its straightness at the junction (Fig. 6d). So the previous ideal model of tube-tube junction can't be applied to understand the 15 formation of peapod derived DWNTs. Here we propose another model that the intra-tube junction should be viewed as a rolled up graphene grain boundary to explain the multiple $5 \mid 7$ formation. In that model, a straight graphene nanoribbon (GNR) is cut along a direction perpendicular to the grain boundary (Fig. 6c) and then 20 been folded into a tube (Fig. $6 c \rightarrow d$ ). The straight GNR edges ensure the straightness of the folded tube (Fig. 6d).

As has been discussed in detail in recent literatures, a $5 \mid 7$ in graphene is an edge dislocation in a $2 \mathrm{D}$ hexagonal lattice and the magnitude of its Burgers vector is $b=0.246 \mathrm{~nm} .{ }^{46}$ In a graphene 25 grain boundary, the requirement of formation energy minimiza-tion aligns all the $5 \mid 7$ pairs linearly. So the mismatch angle between both sides can be estimated by the following equation: ${ }^{47}$

$$
\sin (\Delta \theta)=b / d
$$

where $\Delta \theta$ is the mismatching angle and $d$ is the average distance
30 between neighboring $5 \mid 7$ pairs. As a rolled up grain boundary, the number of $5 \mid 7$ pairs in an intra-tube junction is

$$
N_{5 \mid 7}=l / d=l \times \sin (\Delta \theta) / b \sim l \times \Delta \theta / b
$$

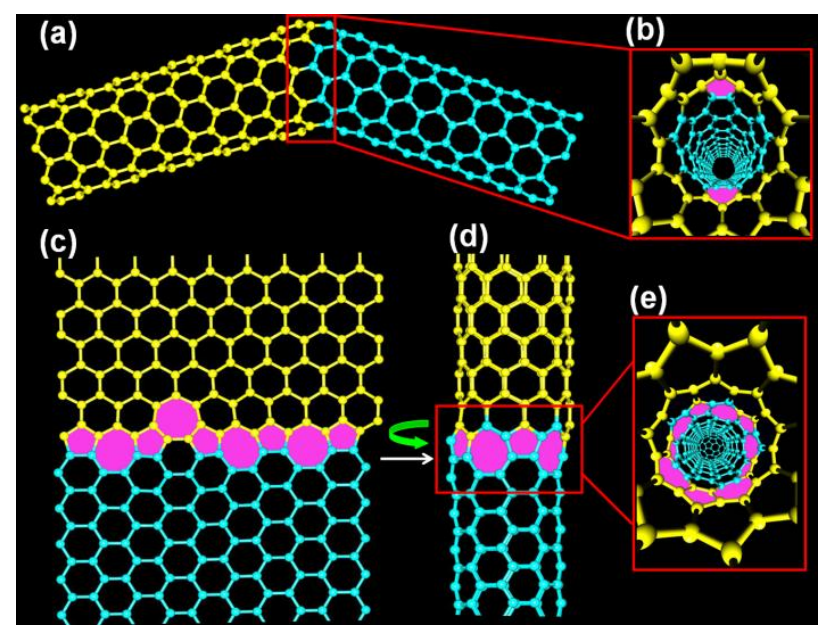

Fig. 6 Two arbitrary tubes can be connected by a pentagon and a 35 heptagon. As an example, a $(9,0)$ tube and a $(5,5)$ tube is connected by separated pentagon and heptagon (a-b) but the tube wall turns a sharp angle of $~ 30$ degree at the intra-tube junction (a). (c) $\rightarrow$ (d) Rolling a graphene grain boundary into a straight tube with an intra-tube junction. The grain boundary contains multiple $5 \mid 7$ pairs forming a ring of 40 topological defects (e). 

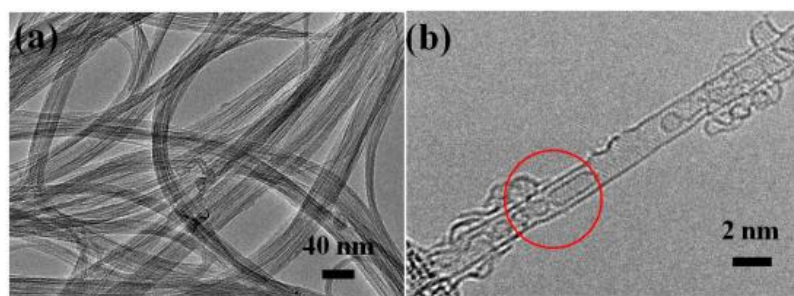

(c)

(d)

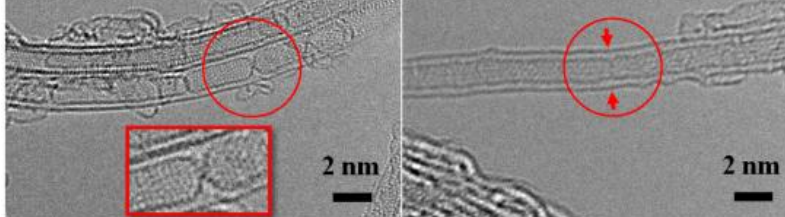

Fig. 7 Transmission electron microscope images of peapod-grown DWNTs that are grown by thermally treating peapods at $1700^{\circ} \mathrm{C}$ in argon using a graphite furnace at (a) low- and (b-d) high-resolutions.

5 where $l$ is the length of tube circumference, $\Delta \theta$ here is the chiral angle difference between the two tube sections. For those inner tubes encapsulated inside a $(18,0)$ SWNT, their diameters are around $0.7 \mathrm{~nm}$. The number of $5 \mid 7$ pairs is $N_{5 \mid 7} \sim 0.15 \times \Delta \theta$ (in degree). For a junction between $(5,5)$ and $(9,0)$ SWNTs, we have 10 $\Delta \theta=30$ degree and $N_{5 \mid 7}=4.5$, which fits the simulated number $N_{5 \mid 7}=5$ perfectly (Fig. $5 \mathrm{~g}$ ).

\subsection{Experimental observation of intra-tube junction}

High-resolution transmission electron microscopy (HRTEM) 15 measurements were taken to examine the coalescence behaviors of inner tubes within a confined space. The peapods derived DWNTs were prepared by thermally treating peapods at 1700 and $1800^{\circ} \mathrm{C}$ in argon (details shown in Experimental Method part). There is no distinctive change in the bundled structure of ${ }_{20}$ peapods-grown DWNTs (Fig. 7a) because the hosting SWNTs are structurally stable up to $2000^{\circ} \mathrm{C}$. In the HRTEM images of DWNTs, the coalescence of short inner tubes with adjacent fullerene (Fig. 7b), the coalescence of adjacent short tubes (Fig. 7c) and the formation of intra-tube junction (Fig. 7d) are clearly 25 seen.

Although the pentagons and heptagons can not be identified near the intra-tube junction because of the resolution limitation of our TEM, the evidence of the intra-tube junction is solid. Thus the experimental observation presents the validity of the 30 theoretical study.

\subsection{A growth model of peapod-grown DWNT}

A model of peapods derived DWNT is shown in Fig. 8 to summarize the study. At the initial nucleation stage, the simultaneous coalescence of many fullerenes in a long carbon 35 peapods results in many isolated short inner tubes of different chiralities inside (Fig. $8 \mathrm{a} \rightarrow \mathrm{b}$ ). These short inner tubes grow longer and longer by exhausting neighboring fullerenes (Fig. 8 $\mathrm{b} \rightarrow \mathrm{c}$ ). Once fullerenes between two inner tubes exhausted, the two tubes contact each other directly and then fuse into one 40 straight tube that contains a stable intra-tube junction (Fig. 8d) The final long inner tube which is formed

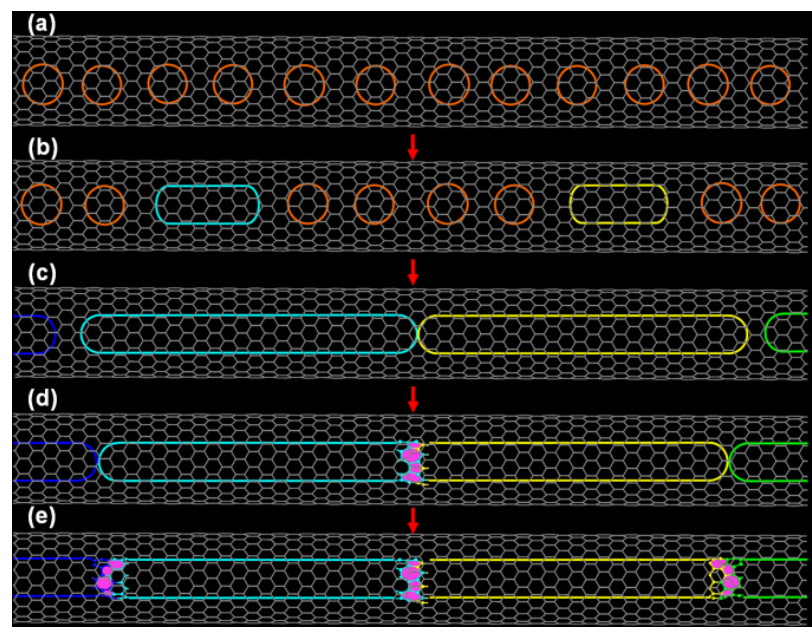

Fig. 8 (a-e)Scheme of the intra-tube junction formation in the inner wall of a peapod derived DWNT. The color of an inner tube of the inner tube 45 section represents its chirality.

by the fusion of many short ones possesses many intra-tube junctions (Fig. 8 e).

\section{Conclusions}

In conclusion, two inner tube elongation processes in the ${ }_{50}$ formation of peapods derived DWNTs are identified by the EDKMC simulation: (i) fusion of tube $+\mathrm{C}_{60}$ and (ii) coalescence of tube + tube. In both processes, only the chirality of very short inner tubes whose stem length less than $2 \mathrm{~nm}$ might be changed and a long inner tube shows its extreme robusticity because of the 55 confinement effect of the outer tube. Our study predicates that the fusion of two long inner tubes must result in a robust intra-tube junction. The formation of the intra-tube junction, which can be viewed as a folded graphene grain boundary, contains multiple pentagon|heptagon pairs (5|7) forming a defect ring. The number 60 of the $5 \mid 7 \mathrm{~s}$ in a junction is proportional to the product of the inner tube's chiral difference and the length of the inner tube circumference. Beyond, careful HRTEM imaging on peapods derived DWNTs samples clearly represents both coalescence processes and shows solid evidence of the intra-tube junction.

\section{${ }_{65}$ Acknowledgements}

We acknowledge the financial support received from Hong Kong Polytechnic University (A-PJ50, A-PH93, A-PD1U). YAK and ME acknowledge the support from the Regional Innovation Cluster Program of Nagano from the MEXT of Japan.

\section{${ }_{70}$ Notes and References}

${ }^{a}$ Institute of Textiles and Clothing, Hong Kong Polytechnic University, Hung Hom, 999077, Hong Kong, E-mail: tcfding@inet.polyu.edu.hk

${ }^{b}$ The Key Laboratory of Liquid Structure and Heredity of Materials, Ministry of Education, Shandong University, Jinan, 250061, People's 75 Republic of China, E-mail: lihuilmy@sdu.edu.cn

${ }^{c}$ Faculty of Engineering, Shinshu University, 4-17-1 Wakasato, Naganoshi, 380-8553, Japan

$\dagger$ Electronic Supplementary Information (ESI) available: experimental details, two tables about the statistics of final inner tubes generated by the 80 EDKMC simulation. See DOI: 10.1039/b000000x/ 
1. B. W. Smith, M. Monthioux and D. E. Luzzi, Nature, 1998, 396, 323-324.

2. B. W. Smith, M. Monthioux and D. E. Luzzi, Chem. Phys. Lett., 1999, 315, 31-36.

5 3. B. W. Smith and D. E. Luzzi, Chem. Phys. Lett., 2000, 321, 169-174.

4. H. Kataura, Y. Maniwa, T. Kodama, K. Kikuchi, K. Hirahara, K. Suenaga, S. Iijima, S. Suzuki, Y. Achiba and W. Kräschmer, Synth. Met., 2001, 121, 1195-1196.

5. X. Liu, T. Pichler, M. Knupfer, M. S. Golden, J. Fink, H. Kataura, Y. Achiba, K. Hirahara and S. Iijima, Phys. Rev. B, 2002, 65, 045419.

6. M. Yudasaka, K. Ajima, K. Suenaga, T. Ichihashi, A. Hashimoto and S. Iijima, Chem. Phys. Lett., 2003, 380, 42-46.

7. H. Kuzmany, R. Pfeiffer, C. Kramberger, T. Pichler, X. Liu, M. Knupfer, J. Fink, H. Kataura, Y. Achiba, B. W. Smith and D. E.

15 Luzzi, Appl. Phys. A, 2003, 76, 449-456.

8. S. Bandow, M. Takizawa, K. Hirahara, M. Yudasaka and S. Iijima, Chem. Phys. Lett., 2001, 337, 48-54.

9. Y. Fujita, N. Niwa, S. Bandow and S. Iijima, Appl. Phys. A, 2006, 85, 307-310.

20 10. R. Pfeiffer, T. Pichler, Y. Kim and H. Kuzmany, Topics Appl. Physics 2008, 111, 495.

11. T. Okazaki, S. Bandow, G. Tamura, Y. Fujita, K. Iakoubovskii, S. Kazaoui, N. Minami, T. Saito, K. Suenaga and S. Iijima, Phys. Rev. B, 2006, 74, 153404.

25 12. M. Koshino, Y. Niimi, E. Nakamura, H. Kataura, T. Okazaki, K. Suenaga and S. Iijima, Nat. Chem., 2010, 2, 117-124.

13. E. Hernandez, V. Meunier, B. W. Smith, R. Rurali, H. Terrones, M Buongiorno Nardelli, M. Terrones, D. E. Luzzi and J. C. Charlier, Nano Lett., 2003, 3, 1037-1042.

30 14. R. Pfeiffer, F. Simon, H. Kuzmany and V. N. Popov, Phys. Rev. B, 2005, 72, 161404.

15. R. Pfeiffer, M. Holzweber, H. Peterlik, H. Kuzmany, Z. Liu, K Suenaga and H. Kataura, Nano Lett., 2007, 7, 2428-2434.

16. H. Kuzmany, W. Plank, R. Pfeiffer and F. Simon, J. Raman Spectrosc. , 2008, 39, 134-140.

17. G. Ning, N. Kishi, H. Okimoto, M. Shiraishi, T. Sugai and H. Shinohara, J. Phys. Chem. C, 2007, 111, 14652-14657.

18. G. G. Samsonidze, R. Saito, N. Kobayashi, A. Gruneis, J. Jiang, A. Jorio, S. G. Chou, G. Dresselhaus and M. S. Dresselhaus, Appl. Phys. 40 Lett., 2004, 85, 5703-5705.

19. H. Muramatsu, T. Hayashi, Y. A. Kim, D. Shimamoto, M. Endo, V. Meunier, B. G. Sumpter, M. Terrones and M. S. Dresselhaus, Small, 2009, 5, 2678-2682.

20. D. Shimamoto, H. Muramatsu, T. Hayashi, Y. A. Kim, M. Endo, J. S.

45 Park, R. Saito, M. Terrones and M. S. Dresselhaus, Appl. Phys. Lett., 2009, 94, 083106-083103.

21. F. Villalpando-Paez, H. Muramatsu, Y. A. Kim, H. Farhat, M. Endo, M. Terrones and M. S. Dresselhaus, Nanoscale, 2010, 2, 406-411.

22. F. Villalpando-Paez, L. G. Moura, C. Fantini, H. Muramatsu, T.

50 Hayashi, Y. A. Kim, M. Endo, M. Terrones, M. A. Pimenta and M. S. Dresselhaus, Phys. Rev. B, 2010, 82, 155416.

23. S. Bandow, T. Hiraoka, T. Yumura, K. Hirahara, H. Shinohara and S. Iijima, Chem. Phys. Lett., 2004, 384, 320-325.

24. J. H. Warner, M. H. Rümmeli, A. Bachmatiuk and B. Büchner, Phys. Rev. $B, 2010, \mathbf{8 1}, 155419$.

25. H. Muramatsu, D. Shimamoto, T. Hayashi, Y. A. Kim, M. Terrones, M. Endo and M. S. Dresselhaus, Adv. Mater., 2011, 23, 1761-1764.

26. H. Su, R. J. Nielsen, A. C. T. van Duin and W. A. Goddard Iii, Phys. Rev. B, 2007, 75, 134107.

60 27. I. Suarez-Martinez, P. J. Higginbottom and N. A. Marks, Carbon, 2010, 48, 3592-3598.

28. Y. Shibuta and S. Maruyama, Heat Tran. Asian Res., 2006, 35, 254264.

29. X. Li, W. Yang and B. Liu, Nano Lett., 2007, 7, 3709-3715.

65 30. A. Trave, F. J. Ribeiro, S. G. Louie and M. L. Cohen, Phys. Rev. B, 2004, 70, 205418.

31. V. Zólyomi, J. Koltai, D. Visontai, L. Oroszlány, Á. Rusznyák, I. László and J. Kürti, Phys. Rev. B, 2010, 82, 195423.

32. A. J. Stone and D. J. Wales, Chem. Phys. Lett., 1986, 128, 501-503.

70 33. Y. Zhao, B. I. Yakobson and R. E. Smalley, Phys. Rev. Lett., 2002, 88, 185501
34. Y. Zhao, R. E. Smalley and B. I. Yakobson, Phys. Rev. B, 2002, 66, 195409.

35. Y. Zhao, Y. Lin and B. I. Yakobson, Phys. Rev. B, 2003, 68, 233403.

75 36. S. Han, M. Yoon, S. Berber, N. Park, E. Osawa, J. Ihm, and D. Tománek Phys. Rev. B, 2004, 70, 113402.

37. I.-H. Lee, S. Jun, H. Kim, S. Y. Kim and Y. Lee, Appl. Phys. Lett., 2006, 88, 011913-011913.

38. F. Ding, Z. W. Xu, B. I. Yakobson, R. J. Young, I. A. Kinloch, S. Cui, L. Deng, P. Puech and M. Monthioux, Phys. Rev. B, 2010, 82, 041403 .

39. F. Ding, K. Jiao, Y. Lin and B. I. Yakobson, Nano Lett., 2007, 7, 681-684

40. F. Ding, K. Jiao, M. Wu and B. I. Yakobson, Phys. Rev. Lett., 2007, 98, 075503 .

41. J. Y. Huang, F. Ding, K. Jiao and B. I. Yakobson, Phys. Rev. Lett., 2007, 99, 175503.

42. K. Hirahara, M. Kociak, S. Bandow, T. Nakahira, K. Itoh, Y. Saito and S. Iijima, Phys. Rev. B, 2006, 73, 195420.

90 43. B. I. Yakobson and F. Ding, ACS Nano, 2011, 5, 1569-1574.

44. R. Saito, G. Dresselhaus, and M. S. Dresselhaus, Phy. Rev. B, 1996, 53, 2044.

45. S. Melchor and J. A. Dobado, J. Chem. Inf. Comput. Sci, 2004, 44, 1639.

95 46. B. I. Yakobson, Appl. Phys. Lett., 1998, 72,918-920.

47. O. V. Yazyev and S. G. Louie, Phys. Rev. B, 2010, 81, 195420. 